\title{
A p-fuzzy model to describe the effects of intervention measures in the spread of the COVID-19 pandemic
}

\author{
Glaucia Maria Bressan ${ }^{1}$ \\ DAMAT/UTFPR, Cornélio Procópio, PR \\ Elenice Weber Stiegelmeier ${ }^{2}$ \\ DAMAT/UTFPR, Cornélio Procópio, PR
}

\begin{abstract}
Resumo. The main purpose of this paper is to analyze how the intervention measures such as lockdown, partial lockdown and no-lockdown help to impede the extent of the severe outbreak of COVID-19. A fuzzy model, considering as input variables, the infected population and the intervention measures and the output variable the level of infestation, was proposed. The numerical results showed that intervention measures play a crucial role in determining the success of COVID19 eradication programs while there is no vaccine available for all the population. Therefore, the model proposed assists government decision making in order to minimize the economic impacts caused by the pandemic.
\end{abstract}

Palavras-chave. Fuzzy model, COVID-19, population dynamics, measure interventions.

\section{Introduction}

The Coronavirus disease, named as COVID-19 pandemic, officially started in 2019 on the Asian continent and has spread widely. Since the emergence of this infectious disease, it has been subsequently span to every continent of the world, except Antarctica [6].

The spread of COVID-19 pandemic has motivated the development of studies and several research activities have been conducted for better understanding the origin, treatments and preventions of this virus [1]. According to [4], it has demanded a quick response from governments in terms of planning contingency efforts that include the imposition of social isolation measures. Even if one or more vaccines have high efficacy and uptake in the population, it will take at least several months for enough people to be vaccinated to confer herd immunity on a population basis [3]. For this reason and for prevent other diseases, the World Health Organization (WHO) [7] recommends that intervention measures, such as personal protective measures (hand hygiene, respiratory etiquette, mask wearing), environmental measures and physical distancing measures, are still necessary, even with the vaccine.

Biomathematical models have been proposed in the literature to describe the dynamics of the COVID-19 epidemic. The paper [4] presents an epidemic model specially tailored for the study of the COVID-19 epidemics. Authors present a case study with three prognostic scenarios for the first wave of the epidemic in the city of Manaus, Brazil. Results show that there are feasible control strategies that could substantially reduce the overload within reasonable time.

Ordinary differential equations are a powerful tool for mathematical modeling of population dynamics, since it is possible to choose the function that determines the variation in relation to

\footnotetext{
${ }^{1}$ glauciabressan@utfpr.edu.br

2 elenicew@utfpr.edu.br
} 
the state of the variable. This fact limits the modeling of many phenomena, since the relationship between state variables and their variations is either subjective or partially known. This modeling is possible using p-fuzzy systems, which incorporate subjective information in both variables and variations and their relationships with variables, making the models more applicable and realistic $[2]$.

By using mathematical models to describe population dynamics and the behavior of epidemics, in the present study, we formulated a mathematical model with intervention measures for COVID19 control and performed a numerical analysis. The interventions considered were the use of social distancing such as lockdown, partial lockdown and no-lockdown. As the purpose of the study is to analyse the effected of intervention strategies adopted, a fuzzy approach was implemented in the model.

\section{Methods}

Let $x$ the population density and $\Delta(x)$ the variation rate, in the iteration $\mathrm{k}$. We consider a discrete p-fuzzy system as differences equation given by

$$
x_{k+1}=x_{k}+\Delta\left(x_{k}\right)
$$

The proposed model has two input variables: the infected-population density and the intervention measures, in a determine period of time, and one output: variation of population $\Delta(x, y)$. Suppose that such terms are modeled by fuzzy sets whose membership functions are obtained from a specialist. We make the following assumptions:

1. The variation of infected populations $\Delta(x)$ depends on the population density of the specie, however, the increase (or decrease) of this rate will also be influenced by seasonality. That is, the population growth rate will change due to the intervention measures adopted.

2. There will be greater growth if no intervention measures are taken and, when these are adopted, the birth rate decreases, reaching the point of having negative growth (only mortality) with a consequent decrease in the number of infected individuals.

3. The state variables, infected population density $(\mathrm{x})$ and intervention measures (y), are represented by the set of linguistic variables: $P_{x}=\{$ low, medium low, medium, medium high, high and highest $\}$ and $M_{y}=$ \{No-lockdown, medium-low, medium-high and total-lockdown . The input variable $y$ will depend on the stage of the interactive system $(k)$.

4. The output variation of infected populations $\Delta(x, y)$, is represented by $L_{\Delta}=$ \{high-negative $\left(H^{-}\right)$, medium-negative $\left(M^{-}\right)$, low-negative $\left(L^{-}\right)$, low-positive $\left(L^{+}\right)$, medium-positive $\left(M^{+}\right)$, high-positive $\left.\left(H^{+}\right)\right\}$.

5. We considered even if the infected population approaches to zero, they may increase again, provided the environment is favorable. That is, we considered that the virus has a latent state and when the condition is favorable, the virus returned.

Figure 1 shows the membership functions of the inputs (Infected population and Intervention measures) and the output (Level of infestation) variables.

Following the assumptions (1)-(5), the system fuzzy rule base is given by 24 rules of the type:

$$
\text { "IF } x \text { is } \alpha_{r} \text { AND the } y \text { is } \beta_{r} \text { THEN } \Delta(x, y) \text { is } \gamma_{r} \text { " }
$$




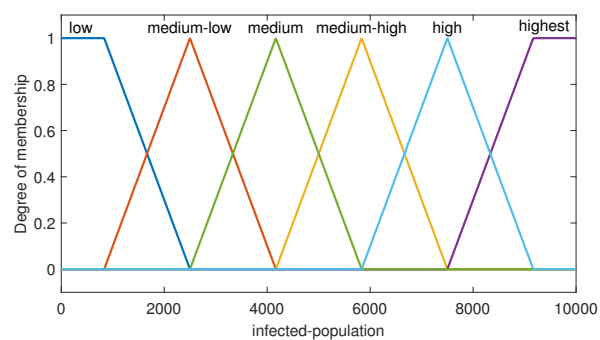

(a)

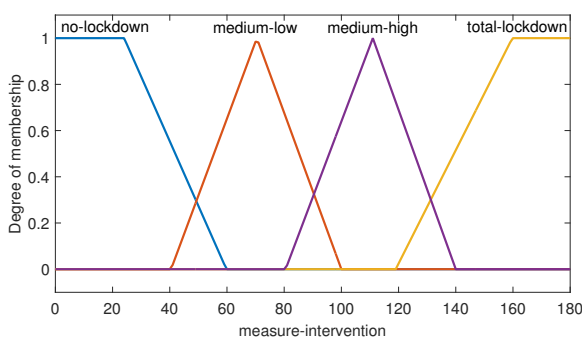

(b)

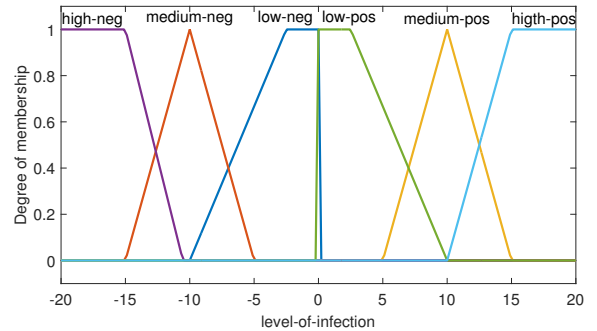

(c)

Figure 1: Input variables: (a) Infected population and (b) Intervention measures. Output variable: (c) Level of infestation

Table 1: Rules base for the p-fuzzy model

\begin{tabular}{lcccc}
\hline & No-lockdown & Medium-low & Medium-high & Total-lockdown \\
\hline Low & $M^{+}$ & $L^{+}$ & $L^{+}$ & $L^{-}$ \\
Medium low & $H^{+}$ & $M^{+}$ & $L^{+}$ & $L^{-}$ \\
Medium & $H^{+}$ & $H^{+}$ & $L^{-}$ & $M^{-}$ \\
Medium high & $H^{+}$ & $M^{+}$ & $M^{-}$ & $M^{-}$ \\
High & $L^{-}$ & $M^{-}$ & $M^{-}$ & $H^{-}$ \\
Highest & $L^{-}$ & $M^{-}$ & $H^{-}$ & $H^{-}$ \\
\hline
\end{tabular}

where $\alpha_{r} \in P_{x}, \beta_{r} \in M_{y}$ and $\gamma_{r} \in L_{\Delta}$ and $r \in\{1 \ldots 24\}$ is the rule considered.

Table 1 shows the 24 linguistic rules constructed, the if-then rules base. We noticed that, if there is a favorable condition for the virus, that is, we active no-lockdown in the environmentaction, so the population growth rate is highest; if the environment is always favorable, so the growth rate is positive, independent of the population size; and an unfavorable environment makes the population variation rate negative (mortality higher than birth).

Therefore, the p-fuzzy model proposed is given by

$$
\left\{\begin{array}{rl}
x_{k+1} & =x_{k}+\Delta\left(x_{k}, y_{k}\right) \\
\left(x_{0}, y_{0}\right) & \in \mathbb{R} \times[0,180] \rightarrow \mathbb{R}
\end{array},\right.
$$

where $\Delta(x, y)$ is given by the rules base shown in Table 1.

In Figure 1 (b), we can see that the measure-intervention has a dominium in $D(y)=[0,180]$, that is, $F: D \rightarrow[0,1]$. Thus, we considered a period of 180 iteration, where in the first 60 iterations, we obtain the membership degree in the no-lockdown set, and after that, in the medium-low and 
high lockdown set and, from 140 iterations, the total-lockdown linguistic variable is activated. In this paper, the time behavior of intervention measures as a cyclical system, since the virus behaves cyclically.

\section{Results and discussion}

We evaluated the dynamics of the infected population considering the intervention measures like no-lockdown, medium low and medium high lockdown and total-lockdown. Here no-lockdown means when no intervention measure is adopted to contain the spread of the virus; medium low and medium high lockdown mean that average low or high measures were adopted by the countries and total-lockdown means social distancing measures taken to hinder the spread [5].

A scenario for the simulation was defined. The initial condition of infected population and the period of time are fixed, $x_{0}=1000$ (daily cases), $t=300$ iterations, respectively. The intervention measures were varied to active each linguist variable in set. The objective is analysed how the intervention measures would be affect the dynamics of the virus. Algorithm 1 describes the main code used to solve the fuzzy model used in the numeric simulation. The initial infected population, $x_{0}$, the intervention measure, $y_{0}$ and $F$ the output of fuzzy rule base are given as input of the Algorithm 1.

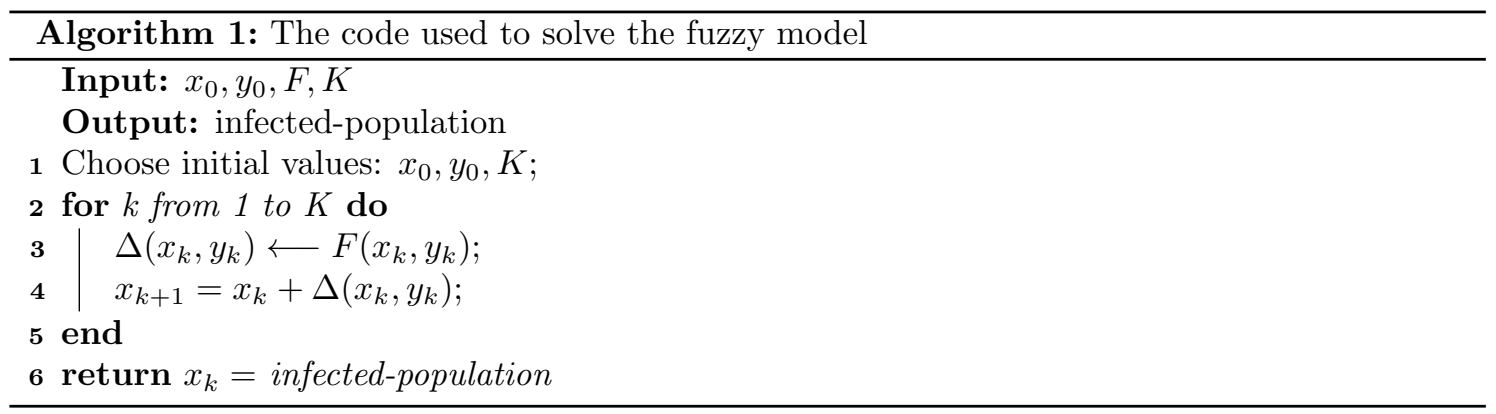

In Fig. 2 we showed the dynamic of infected population varying the initial value of intervention measure. Fig. 2(a) illustrates the evaluated of the infected population considering the scenario where none intervention measure was adopted, so we active the linguist variable no-lockdown. We observe that the infected population grow faster in the begin period and decrease over the time but return to grow when no-lockdown is active again, representing the second wave of virus.

In Fig. 2(b) we active the linguistic variable medium-low lockdown. We observe that there is a slowdown in the number of daily cases but a second wave, around 120 days, appear when the linguistic variable no-lockdown is active again.

Fig. 2(c) considers medium-high lockdown active. So, we have a better scenario with number of daily cases lower. In addition, the model shows the third wave if no intervention measure was adopted around 250 days. Fig. 2(d) illustrates the case where total-lockdown was active. We noticed that a drop occurs in the daily cases during the first days, it occurs due to the high level of intervention adopted (total-lockdown). However, if the population relaxed the measure, the virus returns at higher levels.

The model proposed in this paper offers important insights in controlling the spread of COVID19 using a p-fuzzy approach. The results of the p-fuzzy model were effective since we can understand that the intervention measures are the key to control the level of infected population. Thus, when fuzzy mathematical models are combined with the best intervention measure, the level of infected population can be mitigated. 


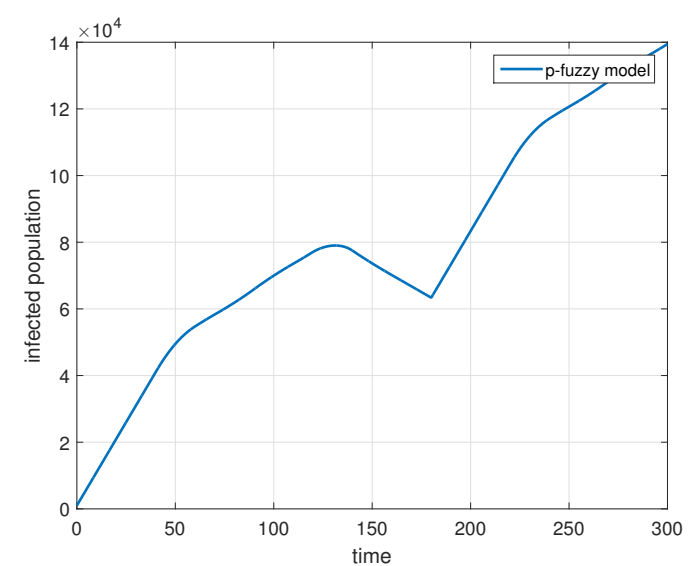

(a)

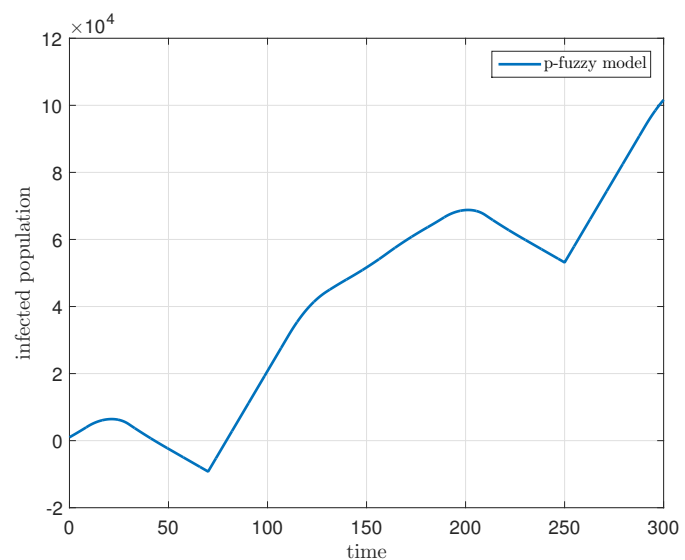

(c)

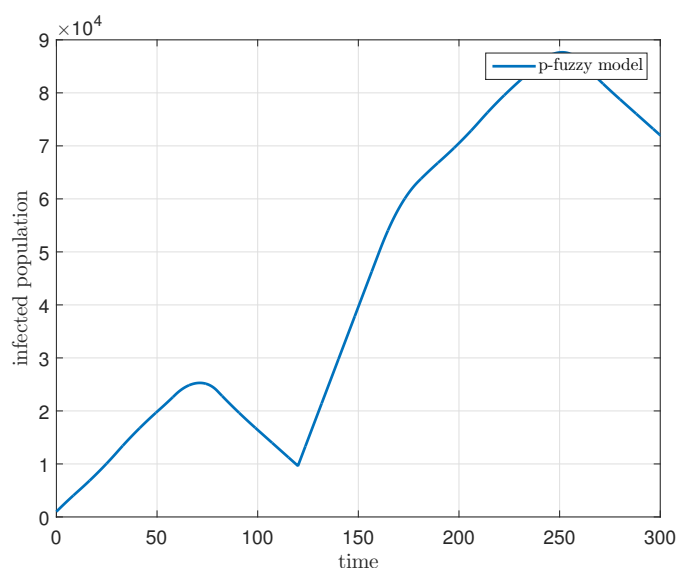

(b)

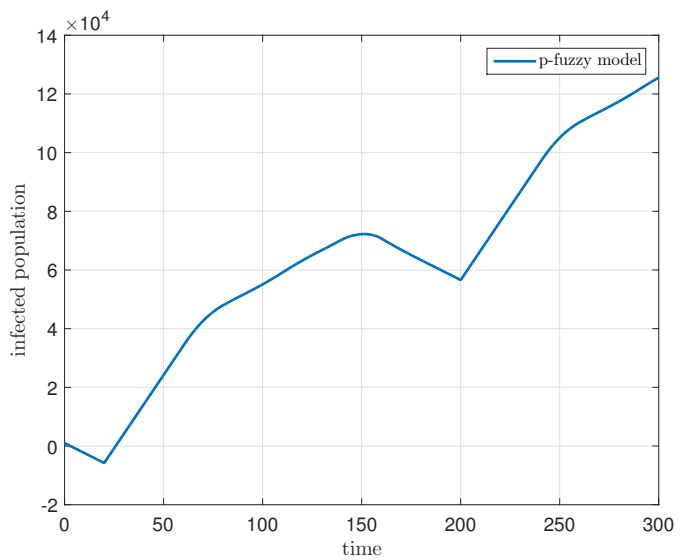

(d)

Figure 2: P-fuzzy model solution with (a) $y_{0}=0$, no-lockdown active; (b) $y_{0}=60$, medium-low lockdown is active; (c) $y_{0}=110$, medium-high lockdown is active and (d) $y_{0}=160$, total-lockdown is active in set $M_{y}$.

\section{Conclusion}

This paper has suggested a fuzzy modelling for the evolution of infected population, focusing on the intervention measures and their effected in the increase of new cases of COVID-19.

The results showed that to consider medium-high lockdown helped to slow down the transmission rates of COVID-19 in the population, however the total lockdown is more effective, while the vaccine is not available for all the population. In addition, the second wave of the pandemic, or the second cycle of the virus, has been more aggressive than the first one and the results presented in this paper demonstrate this fact.

Therefore, the fuzzy model assists government decision making in order to minimize the economic impacts caused by the pandemic and these prevention measures lead to a sudden decline in transmission rate of COVID-19 and turn out to be an effective strategy in containing the virus and saving lives. 


\section{References}

[1] Acter, T., Uddin, N., Das, J., Akhter, A., Choudhury, T. R. and Kim, S. Evolution of severe acute respiratory syndrome coronavirus 2 (SARS-CoV-2) as coronavirus disease 2019 (COVID19) pandemic: A global health emergency. Science of the Total Environment, 730:138996, 2020.

[2] Bassanezi, R. and Barros, L. C. Tópicos de Lógica Fuzzy e Biomatemática. Campinas, IMECC, 2006.

[3] Lerner, A. M., Folkers, G. K. and Fauci, A. S. Preventing the Spread of SARS-CoV-2With Masks and Other "Low-tech" Interventions. American Medical Association, 324(19): 19351936, 2020.

[4] Grzybowski, J. M. V., da Silva, R. V. and Rafikov. M. Expanded SEIRCQ Model Applied to COVID-19 Epidemic Control Strategy Design and Medical Infrastructure Planning. Mathematical Problems in Engineering, vol. 2020, Article ID 8198563, 15 pages, 2020.

[5] Rahaman, M. A., Islam, M. D. S., Khan, A. A., Sarker, B. and Mumtaz, A. Understanding "Quarantine", "Social Distancing", and "Lockdown" during "COVID-19" Pandemic in Response to Global Health: A Conceptual Review. Open Journal of Social Sciences, 8:283-305, 2020.

[6] Rodriguez-Morales, A. J.; Macgregor, K.; Kanagarajah, S.; Patel, D. and Schlagenhauf, P. Going global travel and the 2019 novel coronavirus. Travel medicine and infectious disease, vol. 33, Article 101578, 2020.

[7] World Health Organization (2020, November 4). Considerations for implementing and adjusting public health and social measures in the context of COVID-19. Interim guidance, 1-13, 2020 . 